\title{
Conditioned cardiac and behavioral response topography to an olfactory CS dissociates with age
}

\author{
PAMELA S. HUNT, MAUREEN F. HESS, and BYRON A. CAMPBELL \\ Princeton University, Princeton, New Jersey
}

\begin{abstract}
Conditioned cardiac and behavioral responses to an olfactory stimulus (CS) paired with electric shock (US) were studied in 16-, 23-, and 75-day-old rats. Heart rate and behavior were recorded during a single 15-trial training session in which a 10-sec olfactory stimulus (amyl acetate) was either paired or explicitly unpaired with the US. In the preweanling rats, the conditioned cardiac response consisted of an increase in heart rate (tachycardia) and a slight increase in investigatory behaviors. In contrast, the conditioned cardiac response in the adult rats consisted of a decrease in heart rate (bradycardia) and a striking increase in behavioral immobility (freezing). The 23-day-old rats showed a dichotomous pattern of responding; some showed preweanling-like conditioned responses and some showed adultlike responses. Developmental changes in neural organization and degree of learning are discussed as possible determinants of the transition from infant to adult modes of responding.
\end{abstract}

Qualitative characteristics of a conditioned stimulus (CS), such as stimulus modality, can greatly influence the topography of conditioned behavioral responses in adult animals. Holland (1977) was the first to explicitly describe CS-specific characteristics of appetitive conditioned responses (CRs). When a flashing light $\mathrm{CS}$, which initially elicits a behavioral orienting response characterized primarily by rearing, was repeatedly paired with a food unconditioned stimulus (US), the behavioral CRs during the early portion of the CS period resembled the orienting response to the light (e.g., rearing). In contrast, when an auditory CS was paired with the same food US, the behavioral CR to this stimulus consisted of a prominent head-jerk at CS onset. The head-jerk is a principal characteristic of the orienting response to the auditory stimulus.

Changes in heart rate have been effectively used for studying learning during development, and the direction of the conditioned change in heart rate appears to reflect the modality of the CS. For example, Martin and Alberts (1982) have shown that neonatal rats 5-6 days of age display tachycardia to an olfactory CS previously paired with either lithium chloride or exposure to a cold $\left(10^{\circ} \mathrm{C}\right)$ stressor as the US. Sananes, Gaddy, and Campbell (1988) reported that rat pups 15-21 days of age responded to an olfactory CS paired with a shock US also with heart rate increases (see also Hunt, Hess, \& Campbell, 1994). In contrast, Campbell and Ampuero (1985b) reported bradycardia as the CR to auditory and visual CSs paired with shock. It is interesting that both Sananes et al. (1988) and Campbell and Ampuero (1985b) observed conditioned

This research was supported by NRSA postdoctoral Grant HD07694 to P.S.H., and National Institute of Mental Health Grants MH01562 and MH49496 to B.A.C. Requests for reprints should be addressed to B. A. Campbell, Department of Psychology, Green Hall, Princeton University, Princeton, NJ 08544 (e-mail: bcampbel@pucc.princeton.edu). changes in heart rate in 21-day-old subjects, yet these cardiac responses differed in direction; olfactory cues elicited heart rate increases, whereas auditory cues elicited heart rate decreases. It therefore appears that CS modality may be a critical factor determining the direction of the conditioned cardiac change, at least in the immature rat.

A major limitation in the studies mentioned above examining learned changes in heart rate to an olfactory CS is that the subjects were all preweanling rats, and thus the results cannot necessarily be generalized to apply to older age groups. It may be that the CS specificity of the direction of the heart rate change is restricted to the young animal and may not be similarly determined in the adult. If response topography is the same across development (i.e., tachycardia), this might indicate underlying learning processes that are unique to olfactory information that can be contrasted to those involved in learning about auditory and visual CSs for which the CRs are bradycardia (Campbell \& Ampuero, 1985b; Hunt \& Campbell, in press; Richardson, Wang, \& Campbell, 1995). In contrast, age-related differences in response topography to the olfactory CS would suggest potentially interesting ontogenetic changes in the mode of learning about and/or responding to olfactants. Age-dependent dissociations in CR form to olfactory and gustatory stimuli have been reported previously (Hoffmann, Hunt, \& Spear, 1991; Johanson, Hall, \& Polefrone, 1984). The general purpose of these experiments, therefore, was to determine whether the conditioned heart rate response to an olfactory CS paired with a shock US is acceleratory from infancy to adulthood, or whether there is a change in CR form across ontogeny.

\section{EXPERIMENT 1}

The purpose of Experiment 1 was to compare the topography of conditioned cardiac responses in preweanling 
(16-day-old) and adult rats to an olfactory stimulus paired with a brief footshock. The finding that young rats show tachycardia as the CR to an olfactory CS (Hunt et al., 1994; Martin \& Alberts, 1982; Sananes et al., 1988), but bradycardia as the CR to an auditory CS (Campbell \& Ampuero, 1985b; Richardson et al., 1995), suggests that there is CS specificity in the direction of the conditioned cardiac response. It seemed critical, however, to explore the generality of the conditioned tachycardia across a wider age range than previously examined.

\section{Method}

\section{Subjects}

The subjects were twenty-two 16-day-old and 20 young adult (range, 60-90 days of age) naive Sprague-Dawley-derived rats. The animals were born and reared in the vivarium of the psychology department at Princeton University. Pregnant females were checked daily for births. Two days following birth (day of birth = Postnatal Day 0), the litters were culled to 8 pups each. All animals were raised with the dam and littermates in $48.0 \times 25.5 \times 20.5 \mathrm{~cm}$ polycarbonate maternity cages with wood chips as bedding, and were allowed ad-lib access to Purina Rat Chow and water. On Day 25, the litters were weaned, and the animals were housed in metal group hanging cages with like-sex littermates. The vivarium was maintained on a 16:8-h light:dark cycle, with light onset at $0700 \mathrm{~h}$.

The preweanlings used as subjects were male and female 16-dayold rats; they were removed from the home cage immediately prior to training. No more than 2 pups per litter served in any of the treatment conditions. The adult subjects were males only. Two to 3 days prior to training, these animals were removed from the group cages and placed into individual hanging cages. The subjects were handled for approximately $30 \mathrm{sec}$ per day, and they underwent a minor surgical procedure for implantation of heart rate recording electrodes $20-30 \mathrm{~h}$ prior to training (see below).

\section{Apparatus}

The conditioning chamber was a cylindrical tube, $25 \mathrm{~cm}$ in length and $19 \mathrm{~cm}$ in diameter, made of 0.5 -mm-thick Plexiglas. The cylinder was mounted horizontally in a custom-built sound- attenuating chamber with interior dimensions of $66.0 \times 37.0 \times 81.5 \mathrm{~cm}$. A 4-W white bulb was mounted on an inside wall of the sound-attenuating chamber to provide constant low-level illumination. The bottom of the Plexiglas tube was cut out and fitted with stainless steel bars, $3 \mathrm{~mm}$ in diameter spaced $7 \mathrm{~mm}$ apart (center-to-center). The bars were connected to a constant-current shock generator. The US was a 1sec, 0.6-mA shock, delivered through the grid floor. The olfactory stimulus ( $0.5 \mathrm{ml}$ of amyl acetate diluted in $40 \mathrm{ml}$ of water) was introduced into the cylinder by means of an olfactometer system. The details of this system can be found elsewhere (Hunt, Richardson, Hess, \& Campbell, in press; Sananes et al., 1988). The chamber was heated to $30^{\circ} \mathrm{C}$ for the preweanlings and was maintained at room temperature $\left(21^{\circ}-23^{\circ} \mathrm{C}\right)$ for the adults. Background noise in the chamber was measured at $72 \mathrm{~dB}$ (C scale).

Heart rate was recorded by slightly different means in the preweanling and adult animals. For the 16-day-olds, two transcutaneous steel electrodes, shaped like safety pins, were acutely implanted on the dorsal surface, one at the nape of the neck and the other $1.5 \mathrm{~cm}$ from the base of the tail. Implantation required approximately $10 \mathrm{sec}$ per subject. The electrodes and attached lead wires ( $32 \mathrm{ga}$, Model 2840/7, Alpha Wire Co.) did not appear to interfere with the subjects' movement or general comfort.

For the adults, heart rate was recorded through a pair of surgically implanted electrodes. The electrode assembly was made from a Winchester subminiature connector (Model SM2S) with two 7-cm-long Teflon-coated stainless steel leads (Model AS636, Cooner Wire Co.). The animals were first pretreated with atropine methyl nitrate and were then given an anesthetic dose $(40 \mathrm{mg} / \mathrm{kg}$ ) of sodium pentobarbital (Nembutal; Abbott Laboratories). A patch of skin between the shoulder blades was shaved, and a small incision $(5 \mathrm{~mm})$ was made with a scalpel. The Teflon-coated leads of the electrode were threaded through 20 -ga needles, and the needles were fed through the incision area, just under the animal's skin. When the tips of the needles reached the ventral side, just below the forelegs, the needles and wire leads were pushed through the skin. The needle was taken off and the ends of the wire leads $(5 \mathrm{~mm})$ were stripped of their Teflon coating. The skin around the leads was pulled, allowing the lead to slip back under the skin. The Winchester plug was then sutured in place. Surgery required $5-10$ min per subject. For test, a Winchester subminiature connector (Model SM2P) with lead wires (32-ga, Model 2840/7, Alpha Wire Co.) was attached to the implanted electrode.

Cardiac potentials obtained through both electrodes were amplified by a Grass preamplifier (Model P15). The R-spike activated a Schmitt trigger (Coulbourn Instruments). A microcomputer stored each interbeat interval (IBI), measured to the nearest millisecond. The computer controlled all timing sequences and data collection. The cardiac signal was continuously displayed on a Hitachi oscilloscope (Model V-212).

\section{Procedure}

Prior to training, subjects of each age were divided randomly into one of two groups, designated as paired or unpaired $(n=10-11 /$ group). The electrodes were attached, and the subjects were placed into the conditioning chamber for a 15-min period of acclimation. The subjects assigned to the paired group were given 15 presentations of the $10-\mathrm{sec}$ olfactory stimulus, followed by a $1-\mathrm{sec} 0.6-\mathrm{mA}$ footshock US. The intertrial intervals ranged from 75 to $285 \mathrm{sec}$. The subjects in the unpaired group were treated similarly, except that the US did not occur immediately following the CS. Instead, a random timing program was used to generate a US during each intertrial interval, with the stipulation that a US could not occur either $30 \mathrm{sec}$ before or $30 \mathrm{sec}$ after a CS. Duration of the session, not inclusive of the initial adaptation period, was $59 \mathrm{~min}$.

\section{Treatment of Cardiac Data}

Interbeat intervals were measured during a 5 -sec prestimulus period and during the $10-\mathrm{sec}$ stimulus period on each trial. The IBI data were converted to a beats-per-minute (BPM) measure for analysis. Mean baseline heart rate was subtracted from the heart rate recorded on each of the $10 \mathrm{sec}$ of the stimulus, resulting in a difference score. Negative difference scores indicated cardiac deceleration (bradycardia) during the $\mathrm{CS}$, whereas positive difference scores indicated cardiac acceleration (tachycardia).

\section{Results}

\section{Acquisition of the Heart Rate CR}

Changes from baseline heart rate on each of the $10 \mathrm{sec}$ of the CS were averaged across blocks of three trials, yielding a total of five trial blocks. The data from trial Blocks 1,3 , and 5 were analyzed in a 2 (age) $\times 2$ (conditioning treatment) $\times 3$ (trial blocks) $\times 10$ (seconds) mixed design analysis of variance (ANOVA). In all cases involving a repeated measure, the Geisser-Greenhouse method was used to control for potential inflation in the probability values (Keppel, 1982). The results of the overall ANOVA yielded significant main effects of age, trial blocks, and seconds [smallest $F(9,306)=4.62, p<$ $.05]$. The age $\times$ conditioning treatment, age $\times$ seconds, age $\times$ conditioning treatment $\times$ seconds, and trial blocks $\times$ seconds interactions were also significant [smallest $F(18,612)=5.07, p<.001]$. 
Follow-up ANOVAs and subsequent post hoc comparisons (Newman- Keuls tests; $p=.05$ ) were conducted, and the results are described separately for each age. The data are presented in the top and bottom panels of Figure 1.

Preweanlings. Initially, presentation of the olfactory stimulus elicited a deceleration in heart rate in both paired and unpaired subjects - the typical cardiac orienting response to this stimulus (Hayne, Richardson, \& Campbell, 1991; Hunt et al., 1994). Following pairings of the olfactory CS with footshock, the subjects in the paired group displayed conditioned cardiac acceleration. The cardiac response of the unpaired control group varied nonsignificantly around baseline.

The data presented in the top left panel of Figure 1 shows that the paired and unpaired animals responded identically to the olfactory stimulus on the first trial. The ANOVA conducted on these data yielded a main effect of seconds $[F(9,180)=5.21, p<.01]$, but not of conditioning treatment. By Trial 3, however, the heart rate responses

TAIAL 1 TAIAL 3 TRIAL 7-9 TRIAL 13.15

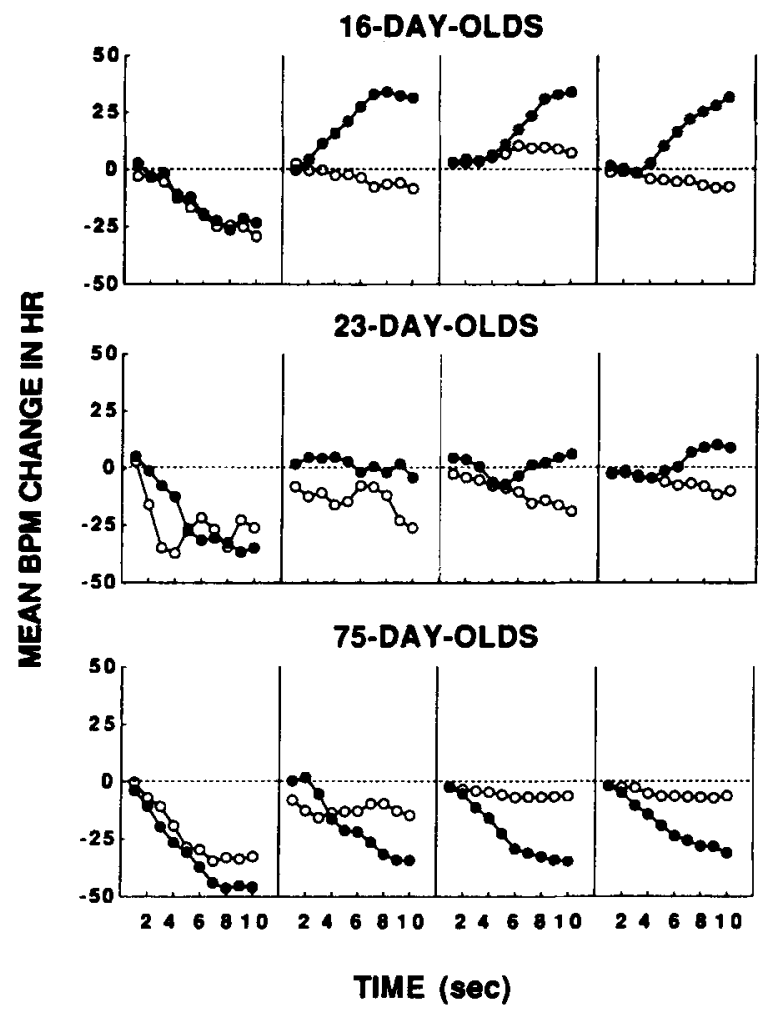

Figure 1. Conditioned cardiac responses to an olfactory CS paired with a footshock US represented as mean beats-per-minute (BPM) change from baseline heart rate on each second of the 10sec CS. The top panel shows the responses of 16-day-olds and the bottom panel the responses of 75-day-olds from Experiment 1. The middle panel shows the responses of 23-day-olds from Experiment 2 . The data plotted are from the first trial (orienting response), the third trial, and the average of Trials 7-9 and 13-15. The filled symbols are the means of the paired CS-US groups, and the open symbols are the means of the explicitly unpaired control groups. All $n s=10-11 /$ group. of these two groups were already differentiated $[F(9,180)$ $=5.93, p<.01]$, and they remained so for Trial Blocks 3 and 5. Contrast analyses conducted on the data from Trial Block 5 confirmed that the tachycardia in the paired group was significantly different from baseline on seconds $6-10$ [smallest $F(10,100)=4.91, p<.05$ ]. The heart rate change of the unpaired group was nonsignificant $[F(10,100)=1.94]$.

Adults. Both the paired and the unpaired groups initially exhibited bradycardia to the novel olfactory CS (cardiac orienting response). Over the course of training, the paired animals displayed conditioned cardiac deceleration, whereas the responses of the unpaired group habituated to near-zero levels. The analysis conducted on the data obtained from Trial 1 indicated that both the paired and the unpaired groups showed equivalent orienting responses to the novel olfactory $\operatorname{CS}[F(1,14)=0.62]$. In contrast to the preweanlings, the paired and unpaired groups were not divergent on Trial $3[F(9,126)=3.03]$; however, the paired and the unpaired groups did differ in their cardiac responses on Trial Blocks 3 and $5[F \mathrm{~s}(9,126)=5.65$ and 5.01, respectively, $p \mathrm{~s}<.01$ ]. Contrast analyses conducted on the Trial Block 5 data indicated that the paired group $[F(10,70)=10.86, p<.001]$, but not the unpaired group $[F(10,70)=1.46]$, showed a significant deviation from baseline.

\section{Baseline Heart Rate}

The 2 (age) $\times 2$ (conditioning treatment) $\times 3$ (trial blocks) mixed design ANOVA conducted on the baseline heart rate data yielded a significant main effect of age $[F(1,34)=96.28, p<.001]$ and a significant age $\times$ conditioning treatment $\times$ trial blocks interaction $[F(2,68)=$ $7.27, p<.01]$. For the 16-day-olds, the baseline heart rates declined in the paired group across the training session, whereas the baselines for the unpaired group remained stable and overall higher than for the paired group. The mean baseline heart rates for the paired and unpaired subjects, respectively, were 515.0 and 529.8 BPM for Trial Block 1; 498.9 and 520.3 BPM for Trial Block 3; and 495.2 and 530.9 BPM for Trial Block 5.

For the adults, the baseline heart rates of the paired group increased during the training session; for the unpaired group, the baseline heart rates decreased. The mean baseline heart rates for the paired and unpaired subjects, respectively, were 415.3 and 443.0 BPM for Trial Block 1; 430.3 and 417.2 BPM for Trial Block 3; and 429.3 and 413.4 BPM for Trial Block 5.

\section{Discussion}

The results of this experiment indicate that preweanling and adult rats respond differently to an olfactory stimulus paired with footshock in terms of the topography of the conditioned cardiac response. The data obtained from the preweanling animals corroborates our earlier findings that these subjects exhibit cardioacceleration to an olfactory CS paired with a shock US (Hunt et al., 1994; Sananes et al., 1988). It appears that a variety of aversive 
USs are capable of supporting learned tachycardia to an olfactory CS in the young animal (see, e.g., Martin \& Alberts, 1982). In contrast to the 16-day-olds, the adults displayed a conditioned deceleratory response to the olfactory CS. Although the conditioning procedure used was identical for the two age groups, the adults showed a direction of cardiac change during the CS opposite to that for the preweanlings. This indicates that the conditioned heart rate response undergoes a developmental transition at some point between 16 days of age and adulthood.

\section{EXPERIMENT 2}

The purpose of Experiment 2 was to examine learned cardiac changes at an age at which the transition in cardiac response topography might reasonably occur. Weanling age subjects ( 23 days old) were trained with the procedures described for Experiment 1. A second purpose was to determine whether topography of the cardiac CR might be related to concurrent changes in somatomotor behavior elicited by the CS. The subjects in this experiment were videotaped during the training session. This allowed for a comparison between the direction of the change in heart rate with changes in behavior elicited by the CS. Several behaviors were chosen for analysis, including sniffing and head lifting, behaviors characteristic of the orienting response to a novel olfactory stimulus (Hayne et al., 1991), and freezing, a species-typical defensive response to fear-eliciting stimuli (Fanselow, 1980; Rudy, 1993).

Method
Subjects
The subjects were twenty-two 23-day-old naive Sprague-Dawley-
derived male and female rats. All animals were reared under the con-
ditions described in Experiment 1. The subjects remained with the
dam prior to training, and no more than 2 animals from any litter
served as subjects in each treatment group.

\section{Apparatus}

The apparatus used for training was the same as that in Experiment 1 . Heart rate was recorded through transcutaneous electrodes, like those used for the 16-day-olds in the previous experiment. In addition, the subjects were videotaped during the training session with a Panasonic VCR (Model AG-6720) and a Panasonic TV camera (Model WV-1854).

\section{Procedure}

Training was identical to that described in Experiment 1. Following a 15-min adaptation period, the subjects in the paired group $(n=11)$ were given 15 odor-footshock pairings. Unpaired subjects $(n=11)$ were given the odor CS and footshock US in an explicitly unpaired manner. Heart rate was recorded for $5 \mathrm{sec}$ before and for the $10 \mathrm{sec}$ during the CS on each trial. Cardiac difference scores were calculated as described for Experiment 1.

In addition, subjects' behavioral responses to the CS were recorded from the videotapes made during the training session. To characterize the behavioral orienting response, behaviors elicited on the first training trial were scored; to characterize the behavioral $\mathrm{CR}$, behaviors elicited on the last three trials (Trial Block 5) were scored. The following behavioral categories were chosen: (1) for- ward locomotion-any movement in a forward direction that was not defined as turning or rearing; (2) turning — circling movements that changed the orientation of the subject without resulting in forward movement; (3) sniffing-a rapid movement of nostrils, typically accompanied by head lifting and usually directed at the odor source; (4) head lifting--movement of the head in an upward direction, with forepaws remaining on the floor; (5) rearing-lifting of the forepaws off the floor, with paws sometimes but not always placed onto the walls of the chamber; (6) freezing - the absence of all movement except that necessary for respiration (Fanselow, 1980). The subjects were observed for $1 \mathrm{sec}$ at each 2 -sec interval during the $10 \mathrm{sec}$ before the CS (pre-CS) and during the 10 -sec CS period, and an immediate judgment of ongoing behavior was made and noted. This yielded five pre-CS and five CS observations for each subject on each trial. With some exception (e.g., freezing), behaviors were not mutually exclusive, allowing for the scoring of more than one behavior during a single observation interval. All behavioral observations were made by a single observer, who was not blind to the training condition. A second observer, unaware of the purpose of the experiment, scored several of the tapes from Experiments 2 and 3 independently. The two observers agreed on $91 \%$ of 600 observations.

\section{Results \\ Acquisition of the Heart Rate CR}

The mean conditioned heart rate response of the 23day-old paired subjects was unstable throughout training and did not clearly reflect the acquisition of a consistent increase or decrease in heart rate. The 2 (conditioning treatment) $\times 3$ (trial blocks) $\times 10$ (seconds) mixed design ANOVA yielded no significant main effects or interactions, although the main effects of conditioning treatment and time, and the trial blocks $\times$ time interaction all approached standard levels of significance [largest $F(1,19)=3.94, .05<p<1.0]$. These data are presented in the middle panels of Figure 1.

\section{Baseline Heart Rate}

The 2 (conditioning treatment) $\times 3$ (trial block) mixed design ANOVA conducted on the baseline heart rate data yielded a significant conditioning treatment $\times$ trial block interaction $[F(2,38)=4.01, p<.05]$. The baseline heart rates of the paired subjects decreased, whereas those of the unpaired subjects increased, across the training session. The mean baseline heart rates for the paired and unpaired subjects, respectively, were 465.7 and 459.0 BPM for Trial Block 1; 455.9 and 483.1 BPM for Trial Block 3; and 451.0 and 488.4 BPM for Trial Block 5.

\section{Comparison of Cardiac and Behavioral CRs}

Although the analyses of the cardiac acquisition data yielded equivocal results, on closer inspection of the data for individual subjects it seemed that the paired subjects were in fact exhibiting learned heart rate responses to the olfactory CS. Of the 11 paired subjects, 6 consistently responded to the CS with cardioacceleration, and 4 others responded with cardiodeceleration (arbitrarily defined as a deviation of greater than 10 BPM from baseline). There was 1 subject that showed unstable responding during the session, responding on some trials with brady- 
cardia and on others with tachycardia. Together, the data from the 11 subjects combined revealed no reliable conditioned cardiac change (Figure 1, middle panels). To further compare the conditioned cardiac and somatomotor responses, we subdivided the paired subjects into two groups: those that showed tachycardia $(n=6)$ and those that showed bradycardia $(n=4)$ on the final trial block of training. Because of the small number of subjects, and the post hoc classification of these subjects into two groups, no statistical analyses were performed on these data.

The mean cardiac change during Trial Block 5 and the associated behavioral responses are shown in Figure 2. The top panels present the responses of the 6 paired subjects showing tachycardia, the middle panels show the responses of the 4 paired subjects displaying bradycardia, and the bottom panels show the responses of the unpaired group (the cardiac data from the unpaired group

\section{TACHYCARDIA}

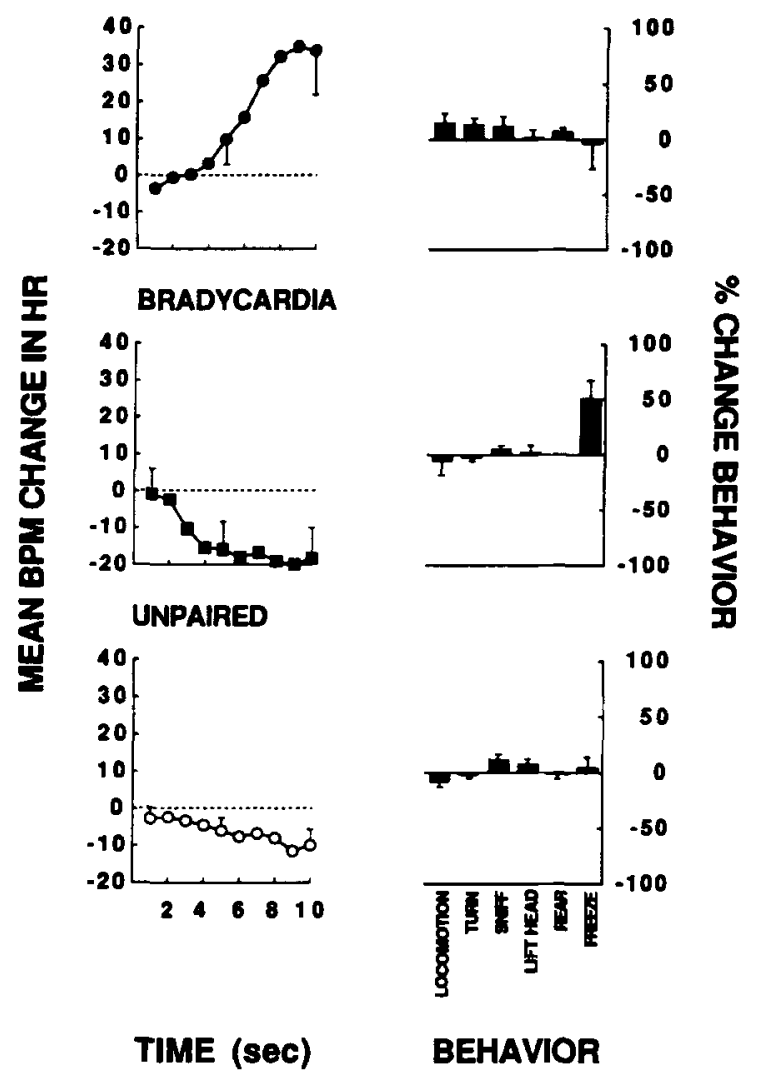

Figure 2. Cardiac responses of 23-day-olds of Experiment 2. Subjects were separated into groups according to their cardiac response at the end of training: paired subjects showing tachycardia as the CR ( $n=6$; top panels); paired subjects exhibiting bradycardia as the CR ( $n=4$; middle panels); and the unpaired control group ( $n=11$; bottom panels). The left-hand panels show the mean ( $\pm S E M$ ) change from baseline heart rate $(H R)$ in beats per minute (BPM) during the 10-sec olfactory stimulus, averaged across the last three acquisition trials. The right-hand panels show the mean $( \pm S E M)$ percent change in each of the six behaviors scored during the 10 -sec CS interval with respect to behaviors recorded during a 10-sec pre-CS interval. shown in Figure 1 has been duplicated here for comparison). The paired subjects that showed conditioned heart rate acceleration exhibited few systematic changes in behavior during the CS period relative to the pre-CS period (Figure 2, top). These subjects responded to the CS with a slight increase in locomotion, turning and sniffing from pre-CS to CS periods. In contrast, the subjects that displayed heart rate deceleration exhibited an increase in freezing during the CS (Figure 2, middle). Finally, the unpaired control group (Figure 2, bottom) exhibited little CS-elicited cardiac or behavioral change.

Correlational analyses were conducted to compare cardiac responses with each of the six behaviors. The peak change in heart rate on Trial Block 5 was correlated with the percent change in each of the behavioral categories across subjects. For the unpaired group, none of the behaviors were significantly correlated with changes in heart rate [largest $r=-.37$ ]. However, for the paired group $(n=11)$, the following behaviors were significantly correlated with peak cardiac change: locomotion $(r=$ $+.61)$, turning $(r=+.69)$, sniffing $(r=+.61)$, and freezing $(r=-.77)$. The correlations for rearing and head lifting were +.24 and +.45 , respectively, and were not statistically significant. The results of these correlational analyses indicate that the change in heart rate at the end of training was related positively to locomotion, turning, and sniffing, but negatively to freezing. ${ }^{1}$

\section{Behavioral Orienting Response}

Subjects responded to the initial olfactory CS presentation with a variety of behaviors classically defined as orienting responses to this stimulus (see Hayne et al., 1991), and these behaviors did not differ between the paired and unpaired subjects. The behaviors seen on Trial 1 included a $32 \%$ increase in head lifting, a $76 \%$ increase in sniffing, and a $17 \%$ increase in rearing to the $\mathrm{CS}$, relative to prestimulus baseline levels.

\section{Discussion}

The results of this experiment suggest, first, that the transition from a disposition for conditioned tachycardia to one toward conditioned bradycardia in response to an olfactory CS paired with a footshock US seems to occur some time around 23 days of age, corresponding to the time of weaning. At this age, some of the subjects in the paired group responded to the olfactory $\mathrm{CS}$ with cardiac acceleration $(n=6)$; others responded with cardiac deceleration $(n=4)$. Second, CS-elicited behaviors were seen to be correlated with directional cardiac changes. Cardiac decreases were associated with an increase in CS-elicited freezing, whereas cardiac increases occurred with a slight increase in investigatory behaviors (locomotion, head lifting, sniffing). These data suggest that animals responding to the aversively conditioned olfactory CS with distinctly age-appropriate cardiac response forms may also show age-specific behavioral responses as well. Alternatively, it may be that the different patterns of behavioral change seen here are evident only during 
this transitional period in development. If, however, the parallel between the direction of the heart rate change and the pattern of CS-elicited behaviors occurs at all ages, it is predicted that adult subjects, showing conditioned bradycardia to the CS, should also respond by freezing during this stimulus. In contrast, preweanlings that reliably display conditioned tachycardia should show an increase in investigatory behaviors toward the CS at the end of training but no CS-elicited freezing. The purpose of the following experiment was to test this prediction.

\section{EXPERIMENT 3}

To test this assumption, 16 - and 75-day-old rats were given 15 pairings of the 10 -sec olfactory stimulus with footshock, and behavioral responses to the CS were recorded. If the direction of the conditioned cardiac response is correlated with a specific pattern of elicited behavioral responses, as was suggested by the results of Experiment 2, it follows that the 16-day-olds should show only orienting-like behaviors during the CS, whereas the adults should exhibit CS-elicited freezing.

\section{Method \\ Subjects \\ The subjects were ten 16-day-old and 10 adult (range, $60-90$ days) naive Sprague-Dawley-derived rats, maintained as those de- scribed in Experiment 1. No more than 2 animals from any litter served as subjects.}

\section{Apparatus}

The apparatus was the same as that described for Experiments 1 and 2.

\section{Procedure}

The procedure for training subjects was the same as that described for the paired groups of Experiments 1 and 2. However, heart rate was not recorded in this study. This avoided the subjects' undergoing the surgical procedure, and also the heart rate responses reported in Experiment 1 have been well documented previously. The session was videotaped, and tapes were later scored for the occurrence of each of the six behaviors during a 10 -sec prestimulus and 10-sec stimulus period, as described for Experiment 2 . The behaviors elicited on the first trial, prior to US presentation, were used to describe the behavioral orienting response to the olfactory CS. The responses observed on the last block of three training trials were used to categorize behavioral conditioned responses. As before, the subjects were observed every other second for $10 \mathrm{sec}$ before CS onset and during the 10-sec CS interval.

\section{Results}

The percent change in the incidence of each of the behaviors exhibited by 16- and 75-day-old subjects on the first trial and the last block of training trials are presented in Figure 3. Each behavior was analyzed separately for each age with Wilcoxon matched-pairs signed-ranks tests for related samples (Siegel, 1956) comparing the percent behavior observed during the stimulus with the percent behavior during the pre-CS period for Trial 1 and Trials 13-15. A nonparametric test was chosen because of the large proportion of zero scores for several of the behav-
TRIAL 1

TRIALS 13-15

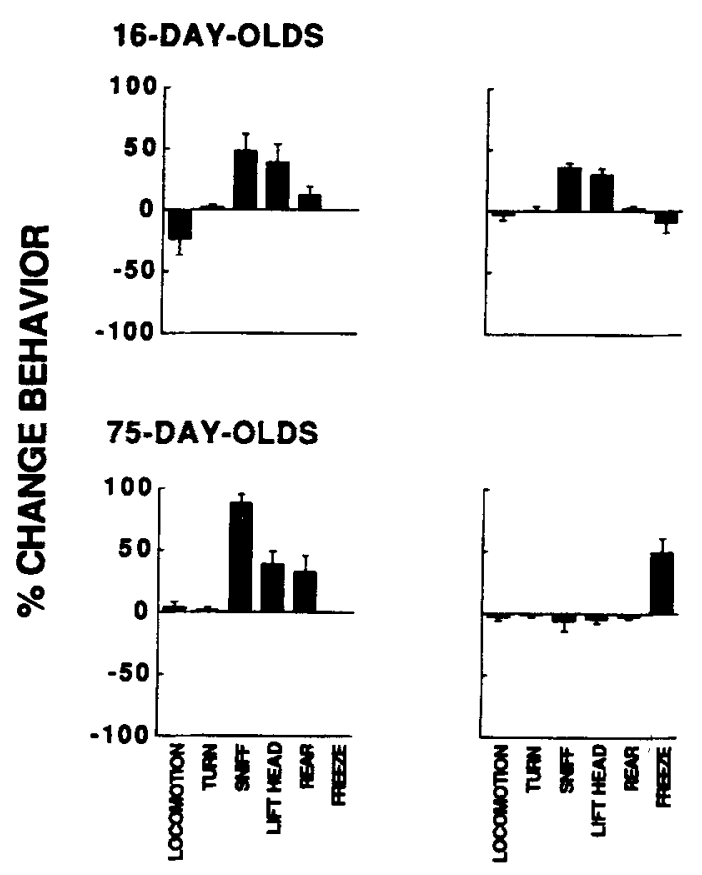

Figure 3. Mean ( $\pm S E M$ ) percent change in each of six behaviors averaged across the $10-\mathrm{sec}$ stimulus interval with respect to behaviors recorded during a 10 -sec pre-CS interval in Experiment 3. Top panels represent the responses observed on Trial 1 (left) and the responses on Trials 13-15 (right) of 16-day-old subjects given paired CS-US trials $(n=10)$. The bottom panels show these responses for 75-day-old subjects given CS-US pairings $(n=10)$.

iors; the Wilcoxon test was chosen specifically, because it is designed for use with within-subjects comparisons.

Behavioral orienting responses were similar at both ages, consisting mainly of increases in sniffing and head lifting (largest $T=3, n=8, p<.05$ ). The changes in the other behaviors were nonsignificant. These responses are depicted in the left panels of Figure 3. The pattern of responses seen at the end of training varied dramatically between the two age groups (Figure 3, right panels). The behaviors exhibited by the 16-day olds on Trials 13-15 did not differ appreciably from those observed on Trial 1 (smallest $T=11, n=9$ ). The prominent CS-elicited behaviors continued to be head lifting and sniffing (largest $T=0, n=10, p<.01)$. In marked contrast, the adults showed a CS-elicited increase in freezing behavior during the $\operatorname{CS}(T=3, n=10, p<.01)$. There were no other significant changes in behavior.

\section{Discussion}

The results of this experiment, together with those of Experiments 1 and 2, suggest that the direction of heart rate change is related to CS-elicited behavioral responses. Conditioned tachycardia (Experiment 1) is associated with behavioral activation in the preweanling rat (Exper- 
iment 3), and conditioned bradycardia (Experiment 1), with behavioral immobility in the adult (Experiment 3 ). Although heart rate was not recorded in this study, the conditioned autonomic responses had been thoroughly established in the preceding experiments. For example, in Experiment 1, 90\% of the 16-day-old subjects responded with conditioned tachycardia, and analogously $90 \%$ of the subjects in this experiment showed a behavioral response pattern of head lifting, sniffing, and no freezing. For the adults, the percentages were similar; $100 \%$ of the adults of Experiment 1 responded with conditioned bradycardia, and $90 \%$ in this experiment responded with an increase in freezing during the CS. It is highly improbable that the subjects in this experiment, either preweanling or adult, were responding with patterns of cardiac changes different from those reported in Experiment 1.

\section{GENERAL DISCUSSION}

The results of these experiments indicate that preweanling rats ( 16 days of age) exhibit cardiac acceleration in response to an olfactory stimulus paired with footshock, whereas adults express cardiac deceleration (Experiment 1). Subjects 23 days of age, as a group, did not show a significant change in heart rate in either direction (Experiment 2). However, 6 of the eleven 23-day-old paired subjects displayed conditioned tachycardia, and another 4 clearly exhibited conditioned bradycardia. These data suggest that there is a transitional stage around weaning, during which the conditioned cardiac response to an olfactory stimulus shifts from conditioned tachycardia to conditioned bradycardia. The direction of change in heart rate during the stimulus period was found to be significantly correlated with patterns of behavioral CRs. In Experiment 2 , the subjects that responded to the CS by freezing were the ones that concurrently expressed conditioned cardiac deceleration. The subjects that continued to express investigatory responses to the CS after CS-US pairings (e.g., sniffing, head lifting) were the ones that showed conditioned increases in heart rate (see also Sananes et al., 1988). To summarize, a clear pattern of cardiac and behavioral change emerged that can be described as follows: The preweanlings showed no qualitative change in CS-elicited behaviors between Trial 1 and Trials 13-15 (Experiment 3 ) but did show a shift from bradycardia to the first few CS presentations to tachycardia on Trials 13-15 (Experiment 1). The adult subjects, however, showed a clear change in behavioral responding to the CS across the training session (Experiment 3), but no change in the form of the cardiac response (Experiment 1). The behavioral orienting responses on the first few trials consisted of sniffing and head lifting, but after CS-US pairings, the response changed to CS-elicited freezing. In these subjects, both the cardiac orienting response and the CR to the olfactory CS were characterized by bradycardia. At 23 days-a transitional age-the subjects tended to divide into two groups: one that showed preweanling-like and another that displayed adult-like cardiac and behavioral patterns of responding.
The reason for the developmental transition in cardiac and behavioral response topography to the olfactory CS is unclear. It is difficult to reconcile these age-dependent response forms in terms of Holland's (1977) characterization of CS-generated CRs (see the introduction). The unconditioned cardiac and behavioral orienting responses did not change appreciably during the course of development. Upon initial presentation of the novel olfactory stimulus, animals of all ages showed cardiac deceleration accompanied by increases in head lifting and sniffing (see also Hayne et al., 1991). The age-related changes in the form of behavioral and cardiac CRs reported here cannot therefore be easily explained in terms of age-specific dispositions toward different orienting responses.

One possible hypothesis is that the neural substrates underlying the cardiac and behavioral CRs change during the course of development. Pertinent to the present data is a recent model of the role of the periaqueductal gray (PAG) in classical fear conditioning proposed by Fanselow and his colleagues (Fanselow, 1991; Fanselow, DeCola, de Oca, \& Landeira-Fernandez, 1995). The lateral aspect of the PAG (IPAG) has been linked to increases in activity (e.g., flight/escape behavior) produced by inescapable footshock (Fanselow et al., 1995; see also Zhang, Bandler, \& Carrive, 1990) and sympathetic nervous system activation (Carrive, 1993; Nakai \& Maeda, 1994). In contrast, expression of conditioned freezing is hypothesized to involve the ventral part of the PAG (vPAG) (Fanselow et al., 1995). According to Fanselow (1991; Fanselow et al., 1995) a CS previously paired with shock comes to activate the VPAG via a projection from the central nucleus of the amygdala. Furthermore, according to this model, if the CS has been strongly conditioned to the US, activation of the IPAG can occur via an excitatory connection between the vPAG and the IPAG. In turn, the IPAG has reciprocal, inhibitory effects back onto the vPAG and the amygdala (Fanselow et al., 1995; Rizvi, Ennis, Behbehani, \& Shipley, 1991).

The application of this model to the pattern of conditioned responding observed in the adult subjects of the present experiments is straightforward. Following pairings of the olfactory CS with shock, the CS comes to activate the vPAG, promoting CS-elicited freezing. The concurrent conditioned bradycardia is probably the result of activation of the parasympathetic nervous system via a projection from the amygdala central nucleus to the dorsal motor nucleus of the vagus and/or nucleus ambiguous (Kapp, Whalen, Supple, \& Pascoe, 1992; McCabe et al., 1992).

This model can be adapted to explain the data obtained from the 16-day-olds if it is assumed that the degree of conditioning to the olfactory CS is stronger in the preweanling animal than in the adult (see, e.g., McKinzie \& Spear, 1995; Wilson \& Sullivan, 1994). This assumption would predict that the strongly conditioned CS would activate the IPAG in the young, but not the older, subjects. The patterns of both autonomic and behavioral responses observed can be explained through the hypothesized activation of the lPAG. The tachycardia seen in re- 
sponse to the CS at this age results primarily from sympathetic nervous system activation (Hunt et al., 1994). The IPAG projects directly to sympathetic premotor neurons in the rostral ventrolateral medulla (Van Bockstaele, Aston-Jones, Pieribone, Ennis, \& Shipley, 1991). Second, activation of the IPAG would prevent the occurrence of conditioned freezing through direct inhibition of the vPAG. For subjects of this age, there was in fact a slight decrease in freezing from pre-CS to CS periods (see Figure 3).

The observed responses of the preweanlings, both cardiac and behavioral, appear to be restricted to the use of an olfactory CS. In the present experiments, the preweanlings responded to the olfactory stimulus with little change in CS-elicited behaviors from the first trial to the last trials of acquisition. It has been shown previously, however, that young animals 15-18 days of age can display substantial CS-elicited freezing in the presence of an auditory CS previously paired with footshock (Rudy, 1993; Rudy \& Morledge, 1994). In contrast, the 16-dayolds in this series of experiments not only displayed a different pattern of conditioned autonomic and behavioral responses to the olfactory CS than the adults, but they also showed a different pattern of conditioned cardiac responses to CSs in different modalities in subjects of similar ages. For example, pairing auditory and visual stimuli with shock elicits conditioned bradycardia in the young rat (Campbell \& Ampuero, 1985b). Thus, the olfactory CS is unique, resulting in distinctive behavioral and cardiac CRs in the preweanling rat. Perhaps the conditioning of a tachycardic defensive response to the olfactory CS, as opposed to the bradycardic (attentional?) response to auditory and visual CSs, has to do with the salience of olfactory stimuli at this age. Since CS saliency can affect rate of acquisition (see, e.g., Rescorla \& Wagner, 1972), a salient olfactory CS may result in more rapid conditioning of defensive behaviors than may a less salient auditory CS. In fact, Campbell and Ampuero (1985a) reported that the cardiac CR to an auditory CS eventually becomes tachycardia in young rats, but only after extended training. According to the present analysis, this shift from bradycardia to tachycardia should be accompanied by a reduction in conditioned freezing.

By this account, then, the different CRs seen across these ages do not result from anatomical maturation or necessarily qualitative differences in learning across these ages, but may instead reflect a quantitative change in the degree of conditioning to olfactory stimuli. Relating proposed age differences in strength of conditioning with activation of distinct anatomical areas in order to explain the different patterns of CR form across ontogeny deserves further empirical attention.

\section{REFERENCES}

Camprell, B. A., \& Ampuero, M. X. (1985a). Conditioned orienting and defensive responses in the developing rat. Infant Behavior \& Development, 8, 425-434.

Campbell, B. A., \& Ampuero, M. X. (1985b). Dissociation of auto- nomic and behavioral components of conditioned fear during development in the rat. Behavioral Neuroscience, 99, 1089-1102.

Carrive, P. (1993). The periaqueductal gray and defensive behavior: Functional representation and neuronal organization. Behavioral Brain Research, 58, 27-47.

FANSELOW, M. S. (1980). Conditional and unconditional components of post-shock freezing. Pavlovian Journal of Biological Sciences, 15, 177-182.

FANSElow, M. S. (1991). The midbrain periaqueductal gray as a coordinator of action in response to fear and anxiety. In A. Depaulis \& R. Bandler (Eds.), The midbrain periaqueductal gray matter: Functional, anatomical, and neurochemical organization (pp. 151-173). New York: Plenum.

Fanselow, M. S., DeCola, J. P., De Oca, B. M., \& LandeiraFERNANDEZ, J. (1995). Ventral and dorsolateral regions of the midbrain periaqueductal gray (PAG) control different stages of defensive behavior: Dorsolateral PAG lesions enhance the defensive freezing produced by massed and immediate shock. Aggressive Behavior, 21, 63-77

Hayne, H., Richardson, R., \& Campbell, B. A. (1991). Developmental constraints on the expression of behavioral and heart-rate orienting responses: $I$. The role of cardiosomatic coupling. Developmental Psychobiology, 24, 1-18.

HoffmanN, H., Hunt, P. S., \& SPEAR, N. E. (1991). Ontogenetic differences in CS palatability following conditioned taste aversion training. Learning \& Motivation, 22, 329-352.

Holland, P. C. (1977). Conditioned stimulus as a determinant of the form of the Pavlovian conditioned response. Journal of Experimental Psychology: Animal Behavior Processes, 3, 77-104.

Hunt, P. S., \& CAMPBELl, B. A. (in press). Developmental dissociation of the components of conditioned fear. In M. E. Bouton \& M. S. Fanselow (Eds.), The functional behaviorism of Robert $C$. Bolles: Learning, motivation, and cognition. Washington, DC: American Psychological Association.

Hunt, P. S., Hess, M. F., \& Campbell, B. A. (1994). Autonomic mediation of unconditioned and conditioned heart rate responses in the 16-day-old rat. Psychobiology, 22, 209-218.

Hunt, P. S., Richardson, R., Hess, M. F., \& Campbell, B. A. (in press). Emergence of conditioned cardiac responses to an olfactory CS paired with an acoustic startle US during development: Form and autonomic origins. Developmental Psychobiology.

Iwata, J., \& LeDoux, J. E. (1988). Dissociation of associative and nonassociative concomitants of classical fear conditioning in freely behaving rat. Behavioral Neuroscience, 102, 66-76.

Johanson, I. B., Hall, W. G., \& Polefrone, J. M. (1984). Appetitive conditioning in neonatal rats: Conditioned ingestive responding to stimuli paired with oral infusions of milk. Developmental Psychobiology, 17, 357-381.

Kapp, B. S., Whalen, P. J., Supple, W. F., \& Pascoe, J. P. (1992). Amygdaloid contributions to conditioned arousal and sensory information processing. In J. P. Aggleton (Ed.), The amygdala: Neurobiological aspects of emotion, memory, and mental dysfunction (pp. 227-254). New York: Wiley-Liss.

KEPPEL, G. (1982). Design and analysis: A researcher's handbook. Englewood Cliffs, NJ: Prentice-Hall.

Martin, L. T., \& Alberts, J. R. (1982). Associative learning in neonatal rats revealed by cardiac response patterns. Journal of Comparative \& Physiological Psychology, 96, 668-675.

McCabe, P. M., Schneiderman, N., Jarrell, T. W., Gentile, C. G., TEICH, A. H., Winters, R. W., \& Liskowsky, D. R. (1992). Central pathways involved in classical differential conditioning of heart rate responses in rabbits. In I. Gormezano \& E. A. Wasserman (Eds.), Learning and memory: The behavioral and biological substrates (pp. 321-346). Hillsdale, NJ: Erlbaum

MCKinzie, D. L., \& SPEAR, N. E. (1995). Ontogenetic differences in conditioning to context and $\mathrm{CS}$ as a function of context saliency and CS-US interval. Animal Learning \& Behavior, 23, 304-313.

NAKAI, M., \& MAEDA, M. (1994). Systemic and regional haemodynamic responses elicited by microinjections of $N$-methyl-D-aspartate into the lateral periaqueductal gray matter in anaesthetized rats. Neuroscience, 58, 777-783. 
RESCORLA, R. A., \& WAGNER, A. R. (1972). A theory of Pavlovian conditioning: Variations in the effectiveness of reinforcement and nonreinforcement. In A. H. Black \& W. F. Prokasy (Eds.), Classical conditioning II: Current research and theory (pp. 64-99). New York: Appleton-Century-Crofts.

Richardson, R., Wang, P., \& Campbell, B. A. (1995). Delayed development of conditioned heart rate responses to auditory stimuli in the rat. Developmental Psychobiology, 28, 221-238.

Rizvi, T. A., Ennis, M., Behbehani, M. M., \& Shipley, M. T. (1991). Connections between the central nucleus of the amygdala and the midbrain periaqueductal gray: Topography and reciprocity. Journal of Comparative Neurology, 303, 121-131.

RuDY, J. W. (1993). Contextual conditioning and auditory cue conditioning dissociate during development. Behavioral Neuroscience, 107, 887-891.

Rudy, J. W., \& MORLEDGe, P. (1994). Ontogeny of contextual fear conditioning in rats: Implications for consolidation, infantile amnesia, and hippocampal system function. Behavioral Neuroscience, 108, 227-234.

Sananes, C. B., Gaddy, J. J., \& Campbell, B. A. (1988). Ontogeny of conditioned heart rate to an olfactory stimulus. Developmental $P_{s y-}$ chobiology, 21, 117-133.

SIEGEL, S. (1956). Nonparametric statistics for the behavioral sciences. New York: McGraw-Hill.

Van Bockstaele, E. J., Aston-Jones, G., Pieribone, V. A., Ennis, M., \& SHIPLEY, M. T. (1991). Subregions of the periaqueductal gray topographically innervate the rostral ventral medulla in the rat. Journal of Comparative Neurology, 309, 305-327.
Wilson, D. A., \& Sullivan, R. M. (1994). Neurobiology of associative learning in the neonate: Early olfactory learning. Behavioral \& Neural Biology, 61, 1-18.

ZhanG, S. P., BANDLER, R., \& Carrive, P. (1990). Flight and immobility evoked by excitatory amino acid microinjection within distinct parts of the subtentorial midbrain periaqueductal gray of the cat. Brain Research, 520, 73-82.

\section{NOTE}

1. Although we are suggesting here that cardiac deceleration co-occurs with freezing, there are data that suggest otherwise. Iwata and LeDoux (1988), for example, report that adult subjects given pairings of a tone CS with footshock later display a pattern of increased heart rate accompanied by CS-elicited freezing. It should be noted, however, that Iwata and LeDoux placed different behavioral constraints on subjects prior to testing for autonomic and behavioral responses. Prior to testing for cardiac changes, the procedure required that the subjects not move during the pre-CS period. In contrast, prior to testing for CS-elicited freezing, a criterion of $15 \mathrm{sec}$ of continuous active exploration of a novel test cage was imposed. Given these differences in pre-CS behaviors, CSelicited heart rate increases and freezing cannot be presumed to necessarily co-occur in other situations, where no motoric restrictions are imposed.

(Manuscript received October 17, 1995; revision accepted for publication April 18, 1996.) 